\title{
"Balancing risk in ophthalmic prescribing: assessing the safety of anti-VEGF therapies and the risks associated with unlicensed medicines"
}

\author{
Bernd Kirchhof
}

Received: 22 July 2012 / Accepted: 23 July 2012 /Published online: 30 August 2012

(C) Springer-Verlag 2012

Kaiser and colleagues are concerned that physicians under pressure from insurance companies or other institutions may prefer unlicensed bevacizumab over ranibizumab. They believe that they convey evidence for comparative lack of efficacy and safety of bevacizumab over ranibizumab. On the other hand-at least in Europe-there is ample marketing effort to foster the prescription of ranibizumab. At the same time, we now have evidence from non-commercial studies on the noninferiority of bevacizumab over ranibizumab (CATT, IVAN [1, 2]), sufficient to license bevacizumab for intraocular use. Since a licence can be filed by pharmaceutical companies only, lack of revenue, common shares between allegedly competing companies, and indifference of national health systems preclude marketing authorization of bevacizumab for neovascular AMD.

The authors aim to fully inform patients, but their evidence is inconclusive or pointless. For example, references 14 and 52-54 do not support "increased risk of bevacizumab compared to ranibizumab", because they date back to between 2001 and 2008. At that time, there were no data available from randomized controlled trials. In principle, not every statistical significant difference is clinically relevant. Even more so, mere "numerical" differences should not be mentioned at all. Thus, if we discuss differences between drugs, we must restrict our attention to statistically significant differences.

\section{B. Kirchhof $(\bowtie)$}

Eye Clinic, University of Cologne,

Kerpener Str. 62,

50937, Cologne, Germany

e-mail: bekirchhof@googlemail.com
For the above-mentioned reasons, the current discussion on differential gain and risks between ranibizumab and bevacizumab must be confined to data from CATT and IVAN, the only studies available with I/A quality of evidence. Since there are no statistical significant differences between comparable treatment groups of bevacizumab and ranibizumab in terms of efficacy, the focus is on safety only.

The known vascular endothelial growth factor related adverse effects, such as myocardial infarction and stroke, do not differ between the drugs.

The only statistically significant differences between ranibizumab and bevacizumab in the CATT study are gastrointestinal disorders: "e.g., haemorrhage, hernia, nausea, and vomiting." Yet according to the label of Avastin $^{\circledR}$ and to a meta-analysis [3], only tumourassociated gastrointestinal perforations and fistulas are specific to bevacizumab. Gastrointestinal perforations and fistulas were reported neither in the CATT study nor in the IVAN study. Kaiser and colleagues point to "the overall significantly higher risk of serious systemic adverse events with bevacizumab versus ranibizumab" in the CATT study. However, this part of the CATT data must be considered as inconclusive, since "when all known vascular endothelial growth factor-related serious adverse events are excluded, most of the imbalance remains, leaving it uncertain whether this difference was the result of chance, imbalances at baseline not captured in multivariate modelling, or truly higher risk" [1].

The authors are mistaken that the study population of IVAN and CATT is too small to reveal individual serious adverse events between treatment groups. The difference in serious adverse events of bevacizumab is obvious in oncology studies with similar study populations compared to the 
CATT study and the IVAN study [3]. Finally, if the study population of CATT and IVAN does not reveal differential risks in serious adverse effects (similar to the Anchor and Marina studies with respect to thromboembolic risks), this qualifies the postulated gastrointestinal risks as rare and of overall low relevance.

In contradiction to Kaiser and colleagues, the evidence from the CATT and IVAN studies signals similar efficacy and safety profiles between ranibizumab and bevacizumab. On these grounds, it is justified to include cost considerations in our prescription behaviour.

\section{References}

1. CATT Research Group (2011) Ranibizumab and bevacizumab for neovascular age-related macular degeneration. $\mathrm{N}$ Engl J Med 364:1897-1908

2. Chakrawvarthy U, Harding SP, Rogers CA, Downes SM, Lotery AJ, Wordsworth S (2012) Ranibizumab versus bevacizumab to treat neovascular age-related macular degenerations. One year findings from the IVAN randomized trial. Ophthalmology 119:1399-1411

3. Hapani S, Shenhong DC (2009) Risk of gastrointestinal perforation in patients with cancer treated with bevacizumab: a metaanalysis. Lancet Oncol 10:599-568 